\title{
An Assessment of Rare Earth Aluminates as Possible Radiation Shields for Artificial Satellites in Low Earth Orbit
}

\author{
Ernesto Ortiz ${ }^{1}$, José Francisco Valdés-Galicia², Rocío García ${ }^{2}$, \\ Lorenzo M. Martínez de la Escalera ${ }^{3}$, Marcos Anzorena ${ }^{2}$, Lorenzo Martínez-Gómez ${ }^{1}$ \\ ${ }^{1}$ Instituto de Ciencias Físicas, Universidad Nacional Autónoma de México, Cuernavaca, Mexico \\ ${ }^{2}$ Instituto de Geofísica, Universidad Nacional Autónoma de México, Ciudad de México, Mexico \\ ${ }^{3}$ Corrosión y Protección (CyP), Ciudad de México, Mexico \\ Email: eortiz@icf.unam.mx
}

How to cite this paper: Ortiz, E., Valdés-Galicia, J.F., García, R., de la Escalera, L.M.M., Anzorena, M. and MartínezGómez, L. (2018) An Assessment of Rare Earth Aluminates as Possible Radiation Shields for Artificial Satellites in Low Earth Orbit. Materials Sciences and Applications, 9, 430-439.

https://doi.org/10.4236/msa.2018.94029

Received: February 7, 2018

Accepted: April 23, 2018

Published: April 26, 2018

Copyright $\odot 2018$ by authors and Scientific Research Publishing Inc. This work is licensed under the Creative Commons Attribution International License (CC BY 4.0).

http://creativecommons.org/licenses/by/4.0/

\begin{abstract}
Charged particles injected into dielectric material of artificial satellites may cause data flipping, command errors and charges in dielectric material properties. In this work we report the results of an evaluation of rare earth aluminates as possible radiation shields for its application in Low Earth Orbit (LEO) satellite construction. With help of Geant 4 software, we calculated the radiation dose that a target receives at a typical LEO $(685 \mathrm{~km})$ as a function of the shield thickness. The target used was a silicon plate, the shields used were hollow cubes of rare earth aluminate walls $\left(\mathrm{YAlO}_{3}, \mathrm{LaAlO}_{3}, \mathrm{NdAlO}_{3}\right.$ and $\left.\mathrm{GdAlO}_{3}\right)$, and we also used aluminium oxide $\left(\mathrm{Al}_{2} \mathrm{O}_{3}\right)$. The radiation source was the measured fluxes of electron and proton with a spectrum corresponding to a LEO. We found that of the total radiation dose received by the target without shield is 5847 microGy/hour, of which, the electrons contribute with $94.9 \%$ and the protons with $5.1 \%$. The rare earth aluminates are a better shield than the $\mathrm{Al}_{2} \mathrm{O}_{3}$ to protect a target against the radiation that permeates a LEO near to equator.
\end{abstract}

\section{Keywords}

Rare Earth Aluminates, Cosmic Radiation, Artificial Satellites

\section{Introduction}

Intense fluxes of magnetospheric electrons (energy $>2 \mathrm{MeV}$ ), are observed while the anomalies were registered at geostationary orbits (high altitude and low inclination) and in the low altitude and high inclination group, but not on high al- 
titude and high inclination orbits. Since this electron population also reaches low altitude in the auroral zones, it produces anomalous behavior in both high altitude and low inclination and low altitude and high inclination satellites, but not in high altitude and high inclination satellites [1] and [2].

Intense fluxes of solar protons (energy $>10 \mathrm{MeV}$ ), are efficient in producing satellite anomalies in the external magnetosphere, particularly relevant for high altitude and high inclination satellites which cross the auroral polar region where solar particles can penetrate more easily. Anomalies due to solar protons are infrequent for high altitude and low inclination group satellites whose arbits are close to the geomagnetic equator [1] and [2].

Recently many efforts have focused on developing dielectric materials with very high quality [3], investigated the dielectric properties of rare earth aluminates, they found that: 1) most rare earth aluminates have suitable permittivities and quality factors for applications as dielectric resonators, and 2) $\mathrm{YAlO}_{3}$ is suggested as a promising substrate material for microstrip antennas utilizing high-temperature superconductor thin films.

Aluminium oxide $\left(\mathrm{Al}_{2} \mathrm{O}_{3}\right)$ is the most widely used material in the engineering application, and it is better than aluminium as radiation shield because the aluminium has a low electron density [4].

With help of Geant4 software, we assess the use of rare earth aluminates as possible radiation shields for artificial satellites in low Earth orbit, for this, we calculed the variation of the radiation dose that electrons (from 1 to $10 \mathrm{MeV}$ ) and protons (from 10 to $500 \mathrm{MeV}$ ) deposit on a silicon plate (target), as a function of the shield thickness.

\section{Setup of the Simulation}

Geant4 software is a toolkit for the simulation of the passage of particles through matter. Geant 4 physics processes cover diverse interactions over an extended energy range, from optical photons and thermal neutrons to the high energy reactions at cosmic ray experiments [5].

In Figure 1, we show schematically the setup for this simulation, it consists of:

1) A silicon plate as a target with a transversal secction of $8 \times 8 \mathrm{~cm}^{2}$ and with a thickness of $1 \mathrm{~mm}$.

2) A hollow cube as shield with a dimension of $(10 \mathrm{~cm})^{3}$ made of a rare earth aluminate: $\mathrm{YAlO}_{3}, \mathrm{LaAlO}_{3}, \mathrm{NdAlO}_{3}$ and $\mathrm{GdAlO}_{3}$. We also used aluminium oxide $\left(\mathrm{Al}_{2} \mathrm{O}_{3}\right)$. The thicknesses of the hollow cubes used were from 0 to $3.6 \mathrm{~mm}$, with increments of $0.1 \mathrm{in} 0.1 \mathrm{~mm}$.

3) A source of: a) electrons with kinetic energy between 1 and $10 \mathrm{MeV}$, and b) protons with kinetic energy between 10 and $500 \mathrm{MeV}$ (see Table 1); these particles were injected isotropically into the target. We used these fluxes because according to [6], are the dominant fluxes for a low Earth orbit $(685 \mathrm{~km})$ and near the equator.

In addition to the fluxes shown in Table 1 , we also include a proton flux (0.3 


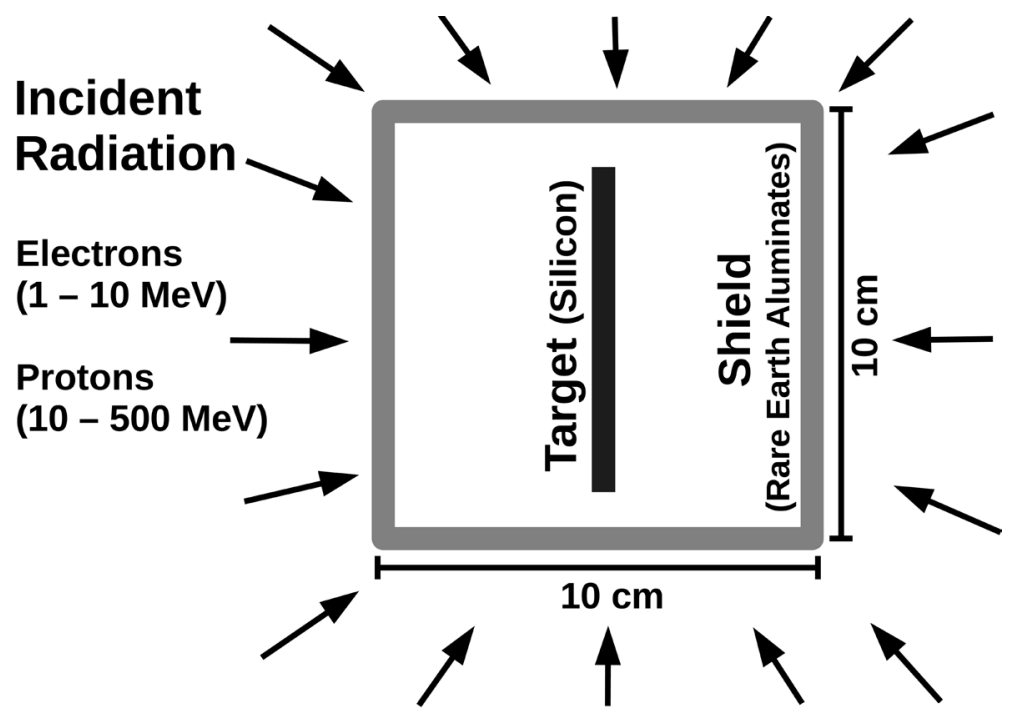

Figure 1. Scheme of the simulation setup. The target is a Silicon plate, the radiation shield is a hollow cube with walls of different thicknesses. The incident radiation are fluxes of electrons and protons. For details see text.

Table 1. Spectrum of electrons and protons for low earth orbit $(685 \mathrm{~km})$ and near the equator, taken from [6].

\begin{tabular}{cccc}
\hline $\begin{array}{c}\text { Electron energy } \\
(\mathrm{MeV})\end{array}$ & $\begin{array}{c}\text { Electron flux } \\
\left(\mathrm{cm}^{-2} \mathrm{~s}^{-1} \mathrm{MeV}^{-1}\right)\end{array}$ & $\begin{array}{c}\text { Proton energy } \\
(\mathrm{MeV})\end{array}$ & $\begin{array}{c}\text { Proton flux } \\
\left(\mathrm{cm}^{-2} \mathrm{~s}^{-1} \mathrm{MeV}^{-1}\right)\end{array}$ \\
\hline 1 & 500 & 10 & 0.180 \\
2 & 461 & 15 & 0.175 \\
3 & 440 & 20 & 0.170 \\
4 & 403 & 25 & 0.120 \\
5 & 325 & 30 & 0.098 \\
6 & 189 & 40 & 0.089 \\
7 & 84 & 50 & 0.081 \\
8 & 31 & 60 & 0.075 \\
9 & 22 & 70 & 0.070 \\
10 & 10 & 80 & 0.064 \\
- & - & 90 & 0.062 \\
- & - & 100 & 0.060 \\
- & - & 200 & 0.080 \\
- & - & 300 & 0.053 \\
- & - & 400 & 0.040 \\
\hline & & 500 & 0.025 \\
\hline
\end{tabular}

$\mathrm{cm}^{-2} \mathrm{~s}^{-1}$ ) with energies between 8 and $12 \mathrm{GeV}$ obtained from [6], this flux represents the galactic cosmic rays (GCR), their spectrum has a maximum at $\sim 10 \mathrm{GeV}$, it presents an abrupt fall for higher energies due to the decrease in the flux of GCR, and it also shows a strong fall for lower energies since GCR with 
energies lower than $10 \mathrm{GeV}$ are modulated by the heliospheric magnetic field.

To estimate with Geant 4 software the real radiation dose (RD) per hour deposited at the target for a low Earth orbit, we injected the particles isotropically into the target, for each material, for each thickness, for each particle species (electron and proton), and for each kinetic energy. We used the particle spectrum reported in [6], see Table 1.

\section{Results and Discussion}

Before presenting the results, we want that the reader to keep in mind that the energy loss mechanism of particles depends on their energy, that is, the dominant process for relativistic particles is bremsstrahlung, which leads them to lose a fraction of their kinetic energy directly proportional to their kinetic energy ( $-\mathrm{dE} / \mathrm{dx}$ proportional to $\mathrm{E})$; whereas for non-relativistic particles the dominant process is ionization, which leads them to lose a fraction of their kinetic energy inversely proportional to their kinetic energy ( $-\mathrm{dE} / \mathrm{dx}$ proportional to $1 / \mathrm{E}$ ).

The electrons used in this work are relativistic particles (see Table 1), then to cross a distance in the material equivalent to a radiation length they will lose a fraction of their energy equivalent to $\sim(1 / \mathrm{e}) \mathrm{E}$. Whereas the protons are nonrelativistic particles, when they cross a material the loss energy rate increases as their kinetic energy decreases, the above occurs until they reach a threshold after which they are stopped.

In Figure 2, we show the radiation dose $(\mathrm{RD})$ deposited at the target by electrons with flat spectrum $\left(10^{6}\right.$ particles per each energy), as a function of the shield thickness of $\mathrm{GdAlO}_{3}$; in this figure we can see that: 1) the RD decreases with increasing of the shield thickness for all energies, and 2) the RD shows a greater attenuation for the electrons of lower energy, and a lower attenuation for

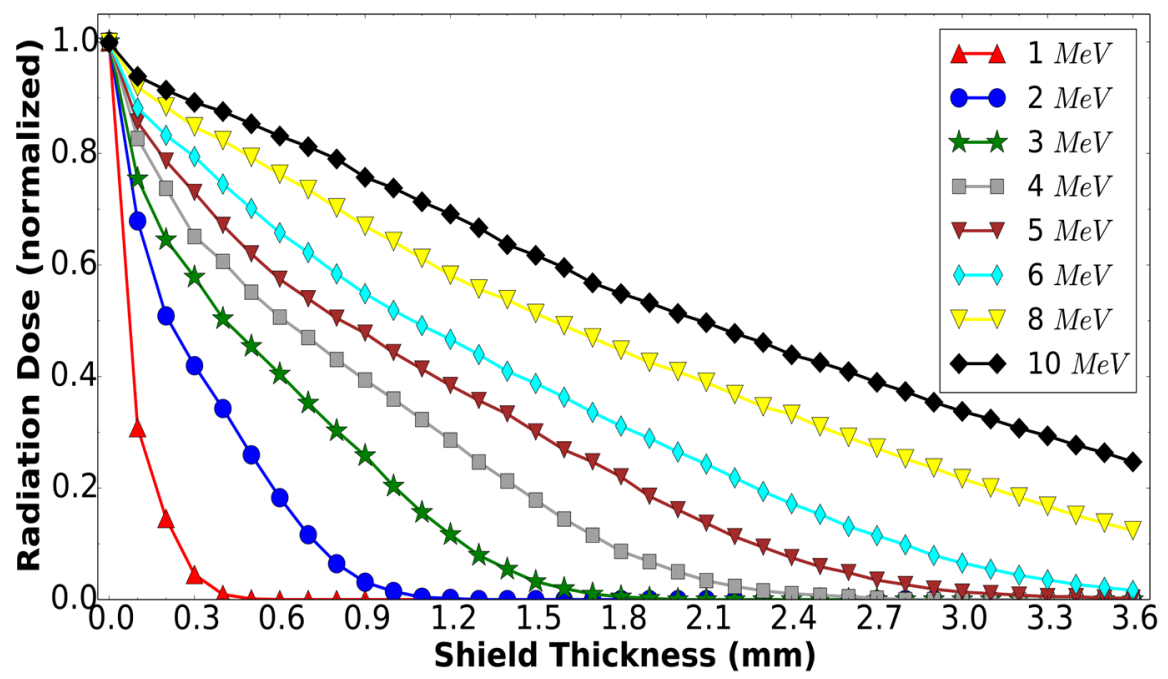

Figure 2. Radiation dose deposited at the target by electrons with flat spectrum, as a function of the shield thickness made of $\mathrm{GdAlO}_{3}$. The RD curves are normalized on 7.56, $4.96,4.19,4.00,3.95,3.92,3.89$ and 3.89 microGy for electrons with 1, 2, 3, 4, 5, 6, 8 and $10 \mathrm{MeV}$ respectively. The electron flux used was $10^{6}$ per each energy. 
the electrons of higher energy. For the remaining materials used as shield, the $\mathrm{RD}$ curves show a similar behavior with a lower attenuation due to the lower density of the materials.

When non-relativistic protons cross a material their loss energy rate is inversely proportional to their kinetic energy; then by increasing the material thickness, the protons lose a greater amount of energy, the above occurs to a limit, after their loss energy rate decreases and they are stopped. The above is named Bragg peak profile [7].

In Figure 3, we show the $\mathrm{RD}$ deposited at the target by protons with flat spectrum $\left(10^{6}\right.$ particles per each energy), as a function of the shield thickness of $\mathrm{GdAlO}_{3}$; in this figure we can see that: 1) the RD deposited by $10 \mathrm{MeV}$ protons decreases abruptly when placing the first shield thicknesses, this curve only shows the descending part of Bragg peak profile; 2) for protons $>10 \mathrm{MeV}$ when increasing the shield thickness, they deposit a greater amount of energy, the above occurs until $0.2,0.6,1.1,1.6$ and $2.9 \mathrm{~mm}$ for protons with 15, 20, 25, 30 and $40 \mathrm{MeV}$ respectively; 3 ) the Bragg peak is generated at a greater shield tickness and with a higher height for higher energy protons.

In Figure 4, we show the $\mathrm{RD}$ deposited at the target by the total set of electrons shown in Table 1 as a function of the shield thickness, in this figure we can see that:

1) For any material used as shield, the RD shows a decrease when increasing the shield thickness.

2) When rare earth aluminates are placed to protect the target, the RD curves show a abrupt decrease from 0 to $\sim 0.6 \mathrm{~mm}$ of shield thickness; this is essentially because the shield thickness is enough to stop a significant amount of low energy electrons, which contribute significantly to the total flux (see Table 1).

3) The RD curves show a less pronounced decrease for shield thicknesses

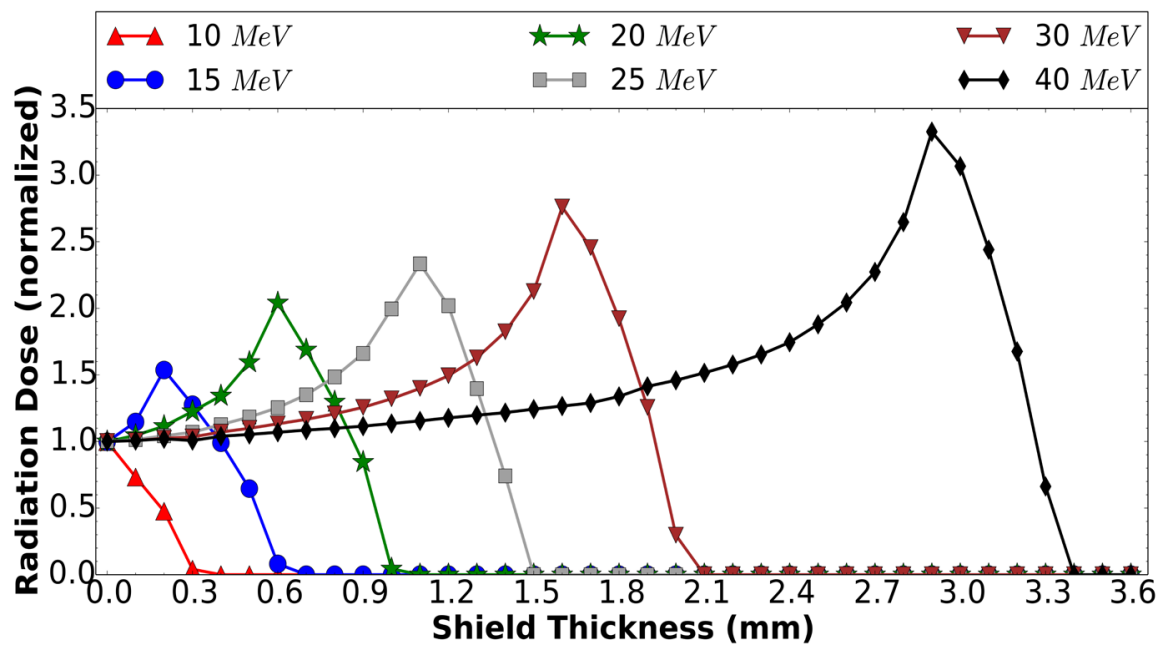

Figure 3. Radiation dose deposited at the target by protons with flat spectrum, as a function of the shield thickness made of $\mathrm{GdAlO}_{3}$. The $\mathrm{RD}$ curves are normalized on 107.4, 78.6, 55.2, 45.5, 38.8 and 30.5 microGy for protons with 10, 15, 20, 25, 30 and $40 \mathrm{MeV}$ respectively. The proton flux used was $10^{6}$ per each energy. 
greater than $0.6 \mathrm{~mm}$, it is because the shield is less efficient for stop high energy electrons.

The $\mathrm{Al}_{2} \mathrm{O}_{3}$ produces attenuation in the $\mathrm{RD}$, however, the rare earth aluminates are better materials to shield since the attenuation of the $\mathrm{RD}$ is considerably larger.

4) From the rare earth aluminates simulated in this work, $\mathrm{GdAlO}_{3}$ generates the largest attenuation in the RD deposited at the target, using a thickness of 0.2 $\mathrm{mm}$ of this material we can reduce the $\mathrm{RD}$ to $\sim 50 \% . \mathrm{Y}_{2} \mathrm{O}_{3}$ is the least efficient material to protect the target against this radiation, a thickness of $0.5 \mathrm{~mm}$ is neccesary to reduce the $\mathrm{RD}$ to $\sim 50 \%$.

In Figure 5, we show the RD deposited at the target by the total set of electrons shown in Table 1 as a function of the shield thickness, in this figure we can see that:

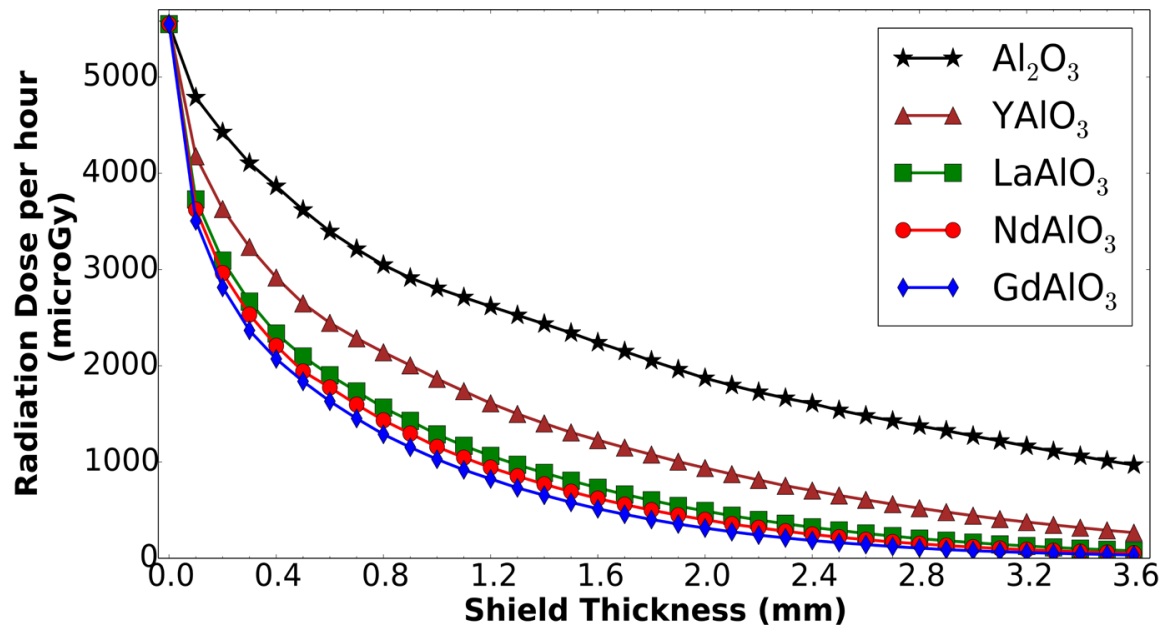

Figure 4. Radiation dose deposited at the target by the total set of electrons shown in $\mathrm{Ta}$ ble 1 , as a function of the shield thickness made of rare earth aluminates. Electron spectrum was taken from [6].

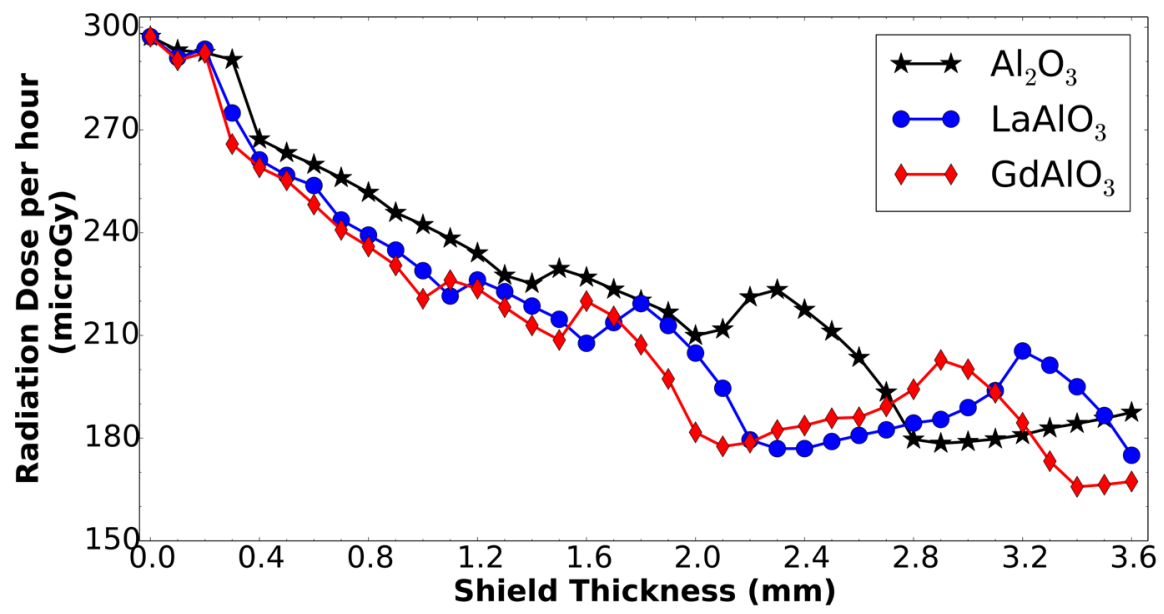

Figure 5. Radiation dose deposited at the target by the total set of protons shown in Table 1 , as a function of the shield thickness made of rare earth aluminates. Proton spectrum was taken from [6]. 
1) For all materials used as shield, the general trend of the RD curves is to decrease with the increase of the shield thickness.

2) The RD curves show an abrupt fall from 0 to $\sim 1.2 \mathrm{~mm}$, the reason for this is that the shield thickness is enough to stop a significant amount of low energy protons $(<25 \mathrm{MeV})$, which contribute significantly to the total RD.

3) After $\sim 1.2 \mathrm{~mm}$ of the shield thickness, the RD curves show some peaks due to the contribution of the Bragg peak generated by each set of protons with a given energy, because we used a discrete spectrum.

4) $\mathrm{GdAlO}_{3}$ is the best material to protect the target against this radiation since it generates a greater attenuation of the $\mathrm{RD}$; graphically we can see that its peaks show a lower height and these are shifted to the left with respect to the peaks of the other materials.

In Figure 6 and Figure 7, we show the total RD deposited at the target by the

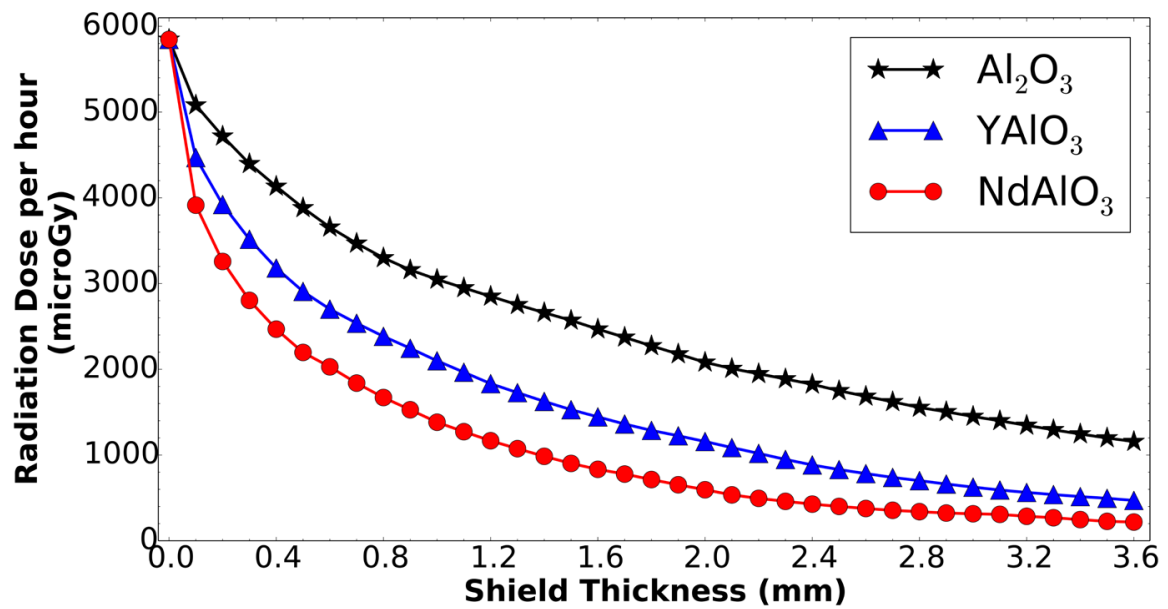

Figure 6. Total radiation dose deposited at the target by the total set of electrons and protons shown in Table 1, as a function of the shield thickness made of $\mathrm{Al}_{2} \mathrm{O}_{3}$ (3.96 $\left.\mathrm{g} / \mathrm{cm}^{3}\right), \mathrm{YAlO}_{3}\left(5.35 \mathrm{~g} / \mathrm{cm}^{3}\right)$ and $\left.\mathrm{NdAlO}_{3}\right)\left(6.91 \mathrm{~g} / \mathrm{cm}^{3}\right)$. Spectrums were taken from [6].

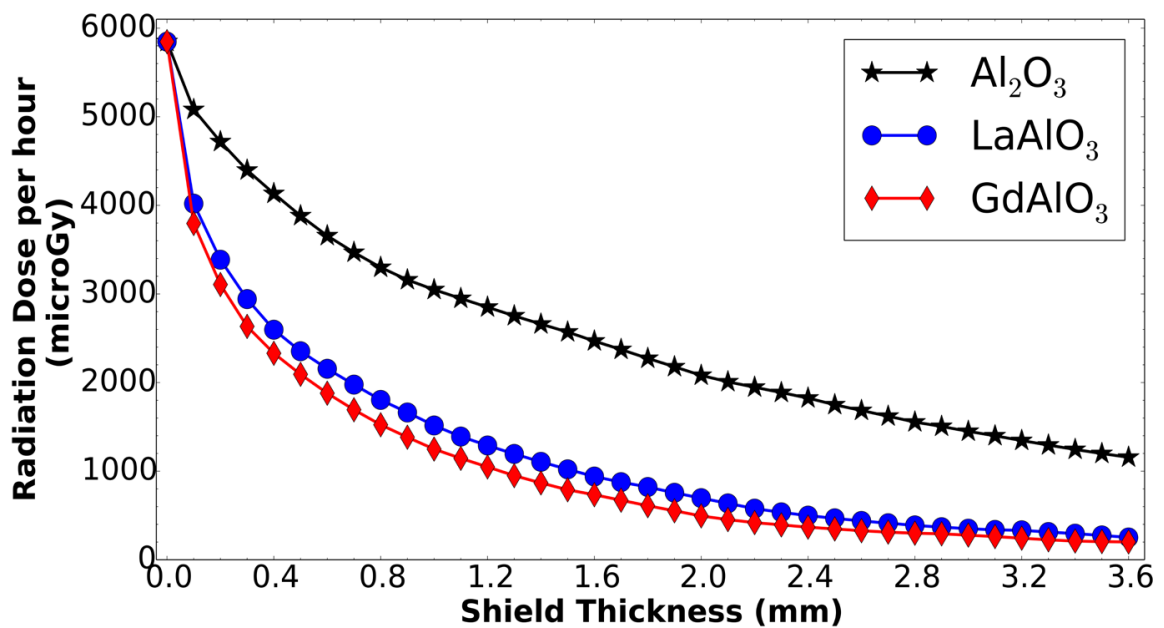

Figure 7. Total radiation dose deposited at the target by the total set of electrons and protons shown in Table 1 , as a function of the shield thickness made of $\mathrm{Al}_{2} \mathrm{O}_{3}$ (3.96 $\left.\mathrm{g} / \mathrm{cm}^{3}\right), \mathrm{LaAlO}_{3}\left(6.53 \mathrm{~g} / \mathrm{cm}^{3}\right)$ and $\mathrm{GdAlO}_{3}\left(7.44 \mathrm{~g} / \mathrm{cm}^{3}\right)$. Spectrums were taken from [6]. 
total set of electrons and protons shown in Table 1, as a function of the shield thickness made of rare earth aluminates and $\mathrm{Al}_{2} \mathrm{O}_{3}$. We present the final results in two different figures to avoid the overlap of the curves. In these figures we can see that:

1) The total $\mathrm{RD}$ deposited at the target without shield is $5847 \mathrm{microGy} / \mathrm{hour}$, of which, 5550 microGy/hour (94.9\%) is by contribution of electrons and 297 microGy/hour (5.1\%) by contribution of protons; the above is due to the fact that electron flux is $\sim 100$ times the proton flux.

2) The $\mathrm{Al}_{2} \mathrm{O}_{3}$ is a good material to shield the target because it produces attenuation in the $\mathrm{RD}$, however, we found that the rare earth aluminates are better materials to shield since the attenuation of the RD is considerably larger.

3) When we used rare earth aluminates as shield, the RD curves show an abrupt decrease from no shield to $0.4 \mathrm{~mm}$ of shield thickness, the RD is reduced to $\sim 50 \%$ compared to the value without shield. This is due to the fact that the shield used is enough to stop a significant amount of electrons with energy $<5 \mathrm{MeV}$ and protons with energy $<15 \mathrm{MeV}$.

4) For thicknesses greater than $0.4 \mathrm{~mm}$ the decrease of the $\mathrm{RD}$ is less pronounced.

When a charged particle penetrates a material, nuclear interactions between the incident particle and the nuclei of the atoms may occur, however, this type of interaction has a very low probability; the dominant process is the Coulomb interactions due to electrical forces between the incident particle and both the electrons and nuclei of the absorbing medium, generating the loss of kinetic energy in the incident particle. In this sense, rare earth aluminates are more efficient materials to reduce the $\mathrm{RD}$ than $\mathrm{Al}_{2} \mathrm{O}_{3}$ because they have a higher density of electrons and nuclei.

The amount of mass is an important factor for artificial satellites. In Table 2, we show the density of the materials used as shield in this work, and the shield thicknesses necessary to reduce the RD deposited at the target to different percentages, in this table we can see that, 1) the ratio between the density of rare earth aluminates and the density of $\mathrm{Al}_{2} \mathrm{O}_{3}$ is less than $1.9 ; 2$ ) the rare earth

Table 2. Shield thicknesses of rare earth aluminates and aluminium oxide, necessary to reduce the RD deposited at the target by total set of electrons and protons shown in Table 1. The $\mathrm{RD}$ deposited at the target without shield is $5,847 \mathrm{microGy} / \mathrm{hour}$. The material densities were taken from [3].

\begin{tabular}{ccccccc}
\hline & & \multicolumn{5}{c}{ Shield Thickness to reduce the RD to: $(\mathrm{mm})$} \\
\cline { 3 - 7 } Shield Material & Density $\left(\mathrm{g} / \mathrm{cm}^{3}\right)$ & $60 \%$ & $50 \%$ & $40 \%$ & $30 \%$ & $20 \%$ \\
\hline $\mathrm{Al}_{2} \mathrm{O}_{3}$ & 3.96 & 0.70 & 1.15 & 1.61 & 2.50 & 3.60 \\
$\mathrm{YAlO}_{3}$ & 5.35 & 0.31 & 0.51 & 0.83 & 1.29 & 2.01 \\
$\mathrm{LaAlO}_{3}$ & 6.53 & 0.19 & 0.31 & 0.50 & 0.83 & 1.35 \\
$\mathrm{NdAlO}_{3}$ & 6.91 & 0.16 & 0.28 & 0.43 & 0.75 & 1.22 \\
$\mathrm{GdAlO}_{3}$ & 7.44 & 0.14 & 0.24 & 0.40 & 0.68 & 1.10 \\
\hline
\end{tabular}


aluminates are an efficient shield since these materials decrease the $\mathrm{RD}$, a reduction that is also possible with $\mathrm{Al}_{2} \mathrm{O}_{3}$, but with a much smaller amount of mass.

In Figure 6 and Figure 7, we omited the contribution of the galactic cosmic rays because this is not very important, the $\mathrm{RD}$ that they deposit at the target is low ( $\sim 1 \mathrm{microGy} / \mathrm{hour})$ and does not present variations with the different thicknesses used since these particles have high energy $(\sim 10 \mathrm{GeV})$.

\section{Summary}

This study is aimed to assess of rare earth aluminates as possible radiation shield for artificial satellites in low Earth orbit. To do this work, we used Geant4 software to calculate the radiation dose that electrons (from 1 to $10 \mathrm{MeV}$ ) and protons (from 10 to $500 \mathrm{MeV}$ ) deposit at a silicon plate as a function of the thickness of the hollow cubes made with rare earth aluminates and aluminium oxide used as shield. According to [6], these particles with these energies are the dominant fluxes in a low Earth orbit near from equator.

In this work, we found that the total radiation dose received by the target without shield is 5847 microGy/hour, which the electrons contribute with $94.9 \%$ and the protons with $5.1 \%$.

We found that the rare earth aluminates used in this work, provide better protection against radiation (see Table 1), a protection that is also possible with $\mathrm{Al}_{2} \mathrm{O}_{3}$, but with a much smaller amount of mass. We conclude that $\mathrm{NdAlO}_{3}$ and $\mathrm{GdAlO}_{3}$ are the most efficient shield for spacecrafts in low Earth orbit.

The placing in orbit of an artificial satellite involves many variables for the materials; in a future work, we will assess the rare earth aluminates under mechanical and thermal tests to which the artificial satellites are subjected during their launching.

\section{Acknowledgements}

The authors acknowledge the support the Fondo CONACYT-SENER-Sustentabilidad Energética Project 232611.

\section{References}

[1] Dorman, L.I., Belov, A.V., Eroshenko, E.A., Gromova, L.I., Iucci, N., Levitin, A.E., Parisi, M., Ptitsyna, N.G., Pustil'nik, L.A., Tyasto, M.I., Vernova, E.S., Villoresi, G., Yanke, V.G. and Zukerman, I.G. (2005) Different Space Weather Effects in Anomalies of the High and Low Orbital Satellites. Advances in Space Research, 36, 2530-2536. https://doi.org/10.1016/j.asr.2004.05.007

[2] Iucci, N., Dorman, L.I., Levitin, A.E., Belov, A.V., Eroshenko, E.A., Ptitsyna, N.G., Villoresi, G., Chizhenkov, G.V., Gromova, L.I., Parisi, M., Tyasto, M.I. and Yanke, V.G. (2006) Spacecraft Operational Anomalies and Space Weather Impact Hazards. Advances in Space Research, 37, 184-190. https://doi.org/10.1016/j.asr.2005.03.028

[3] Cho, S.-Y., Kim, I.-T. and Hong, K.S. (1999) Microwave Dielectric Propieties and Applications of Rare-Earths Aluminates. Journal of Materials Research, 14.

[4] Capali, V., Yesil, T.A., Kaya, G., Kaplan, A., Yavuz, M. and Tilki, T. (2015) Geant4 Calculations for Space Radiation Shielding Material $\mathrm{Al}_{2} \mathrm{O}_{3}$. EPJ Web of Conferences, 
100, 02002. https://doi.org/10.1051/epjconf/201510002002

[5] Allison, J., Amako, K., Apostolakis, J., Arce, P., Asai, M., Aso, T., et al. (2016) Recent Developments in Geant4. Nuclear Instruments and Methods in Physics Research $A, 835,186-225$. https://doi.org/10.1016/j.nima.2016.06.125

[6] Suparta, W. and Zulkeple, S.K. (2014) Spatial Analysis of Galactic Cosmic Ray Particles in Low Earth Orbit/Near Equator Orbit Using SPENVIS. Journal of Physics: Conference Series, 495, 012040.

http://iopscience.iop.org/article/10.1088/1742-6596/495/1/012040/pdf https://doi.org/10.1088/1742-6596/495/1/012040

[7] Cirrone, G.A.P., Cuttone, G., Di Rosa, F., Guatelli, S., Mascialino, B., Pia, M.G. and Russo, G. (2006) Validation of Geant4 Physics Models for tha Simulation of the Proton Bragg Peak. IEEE Nuclear Science Symposium Conference Record, 788-792. 